\title{
Polynomial Subspace Decomposition for Broadband Angle of Arrival Estimation
}

\author{
Mohamed A. Alrmah, Jamie Corr, Ahmed Alzin, Keith Thompson, and Stephan Weiss \\ Department of Electronic \& Electrical Engineering, University of Strathclyde, Glasgow, Scotland \\ \{mohamed.alrmah,jamie.corr, ahmed.alzin, keith.thompson,stephan.weiss\}@ strath.ac.uk
}

\begin{abstract}
In this paper we study the impact of polynomial or broadband subspace decompositions on any subsequent processing, which here uses the example of a broadband angle of arrival estimation technique using a recently proposed polynomial MUSIC (P-MUSIC) algorithm. The subspace decompositions are performed by iterative polynomial EVDs, which differ in their approximations to diagonalise and spectrally majorise $s$ apce-time covariance matrix. We here show that a better diagonalisation has a significant impact on the accuracy of defining broadband signal and noise subspaces, demonstrated by a much higher accuracy of the P-MUSIC spectrum.
\end{abstract}

\section{INTRODUCTION}

For broadband angle of arrival (AoA) estimation, powerful narrowband methods such as the multiple signal classification (MUSIC) algorithm [5] are not directly applicable, and approaches e.g. based on performing MUSIC in independent frequency bins are likely to result in poor performance, particularly if signal frequencies do not coincide with frequency bins [1].

Amongst dedicated broadband AoA estimation algorithms, the coherent signal subspace method (CSSM) [7] combines covariance matrices at different frequency bins coherently by means of focussing matrices whose determination has most recently been addressed by an auto-focussing approach in [9]. A parameterised spatial covariance (PSC) approach [2], [6] scans for possible AoAs using a proper broadband approach, but is only suitable at resolving single AoA. In [1], we have exploited a polynomial matrix decomposition in [3] to generalise MUSIC to the case of spatio-temporal polynomial covariance matrices. The purpose of this paper is to highlight the accuracy of the P-MUSIC by exploiting the impact of the performance of different polynomial matrix decomposition techniques in [3], [4].

In this paper, we analyse the impact of the decomposition techniques for the polynomial space-time covariance matrix on the performance of the polynomial MUSIC algorithm introduced in [1]. To accomplish this, Sec. V reviews different approaches for implementing a polynomial eigenvalue decomposition such as the second order sequential best rotation algorithm (SBR2) and the multiple shift maximum element sequential matrix diagonalisation algorithm (MSME-SMD). Sec. II introduces the data model, with narrow and broadband approaches to AoA approaches outlined in Secs. III and IV. Simulation results are provided in Sec. VI to demonstrate and compare the accuracy of our proposed P-MUSIC approach based on a PEVD utilising SBR2 or MSME-SMD. Conclusions are drawn in Sec. VII.

Notation. Matrix and vector quantities are represented by upper and lowercase bold face variables, e.g. A and a. The Hermitian transpose of $\mathbf{A}$ is denoted as $\mathbf{A}^{\mathrm{H}}$. Polynomial vectors and matrices are written as $\boldsymbol{a}(z)$ and $\boldsymbol{A}(z)$, with the parahermitian $\tilde{\boldsymbol{A}}(z)=\boldsymbol{A}^{\mathrm{H}}\left(z^{-1}\right)$. A transform pair $a[n]$ and $A(z)=\sum_{n=-\infty}^{\infty} a[n] z^{-n}$ is abbreviated as $a[n] \circ \longrightarrow A(z)$.

\section{Broadband ARray Data Model}

\section{A. Data Model}

Multichannel data from an $M$-element array is collected in a vector $\mathbf{x}[n] \in \mathbb{C}^{M}$. We assume that $J$ far-field sources illuminate the array and contribute to $\mathbf{x}[n]$ in addition to isotropic white noise $\mathbf{v}[n]$,

$$
\mathbf{x}[n]=\sum_{j=1}^{J} \mathbf{s}_{j}[n]=\sum_{j=1}^{J} \sum_{\nu} \mathbf{a}_{j}[\nu-n] s_{j}[\nu]+\mathbf{v}[n]
$$

where $s_{j}[n]$ is the $j$ th source signal, $\mathbf{s}_{j}[n]$ its projection onto the array, and $\mathbf{a}_{j}[n]$ the corresponding broadband steering vector, forming the contribution of the $j$ th source to the array. This model only considers the angle of arrival, but neglects any attenuation in the medium.

\section{B. Broadband Steering Vector}

For an arbitrary array configuration, where $\mathbf{r}_{m}$ describes the coordinates of the $m$ th array element, the broadband steering vector consists of delays

$$
\mathbf{a}_{j}[n]=\left[\begin{array}{c}
\delta\left[n-\tau_{j, 0}\right] \\
\vdots \\
\delta\left[n-\tau_{j, M-1}\right]
\end{array}\right],
$$

with the time delay

$$
\tau_{j, m}=\frac{\mathbf{k}_{j}^{\mathrm{H}} \mathbf{r}_{m}}{c T_{\mathrm{s}}}
$$

in samples. The slowness vector $\mathbf{k}_{j}$ is orthogonal to the planar wave front emanating from the $j$ th source, with $c$ the propagation speed in the medium and $T_{\mathrm{s}}$ the sampling period. The noise $\mathbf{v}[n]$ is assumed to be independent and identically distributed, such that $\mathcal{E}\left\{\mathbf{v}[n] \mathbf{v}^{\mathrm{H}}[n-\tau]\right\}=\delta[\tau] \sigma_{v}^{2} \mathbf{I}$. 


\section{Narrowband Steering Vector}

For $\mathbf{s}_{j}[n]$ in (1) describing the contribution from the $j$ th source to $\mathbf{x}[n]$, the first sensor signal can be taken as reference, and the relative delays of the remaining sensor signals can be characterised as

$$
\mathbf{s}_{j}[n]=\left[\begin{array}{c}
s_{j}[n] \\
s_{j}\left[n-\Delta \tau_{j, 1}\right] \\
\vdots \\
s_{j}\left[n-\Delta \tau_{j, M-1}\right]
\end{array}\right],
$$

with $\Delta \tau_{j, m}=\tau_{j, m}-\tau_{j, 0}$. For a narrowband source with normalised angular frequency $\Omega$ and a reference signal $s_{j}[n]=$ $e^{j \Omega n}$, the time delays $\Delta \tau_{j, m}$ collapse to simple phase shifts

$$
\mathbf{s}_{j}[n]=\left[\begin{array}{c}
1 \\
e^{-j \Omega \Delta \tau_{, 1}} \\
\vdots \\
e^{-j \Omega \Delta \tau_{j, M-1}}
\end{array}\right] e^{j \Omega n}=\mathbf{a}_{\Omega, \vartheta_{j}} e^{j \Omega n}
$$

where $\mathbf{a}_{\Omega, \vartheta_{k}}$ is termed the narrowband steering vector.

\section{NARRowband Subspace DeCOMPOSITION}

\section{A. Narrowband Covariance Matrix}

For the narrowband case, with $J$ sources $s_{j}[n]$ characterised by pairs $\left\{\Omega_{j}, \vartheta_{j}\right\}$ the array vector in (1) simplifies to

$$
\mathbf{x}[n]=\sum_{l=1}^{L} \mathbf{a}_{\Omega_{j}, \vartheta_{j}} s_{j}[n]+\mathbf{v}[n] .
$$

The covariance matrix for this narrowband scenario only needs to capture instantaneous correlation, such that $\mathbf{R}=$ $\mathcal{E}\left\{\mathbf{x}[n] \mathbf{x}^{\mathrm{H}}[n]\right\} \in \mathbb{C}^{M \times M}$, with $\mathcal{E}\{\cdot\}$ the expectation operator, sufficiently describes the array's second order statistics. In the case of $J$ uncorrelated and mutually independent source signals with power $\sigma_{j}^{2}, j \in(1, J)$,

$$
\mathbf{R}=\sum_{j=1}^{J} \sigma_{j}^{2} \mathbf{a}_{\Omega, \vartheta_{j}} \mathbf{a}_{\Omega, \vartheta_{j}}^{\mathrm{H}}+\sigma_{v}^{2} \mathbf{I}
$$

The maximum rank of $\mathbf{R}, \operatorname{rank}\{\mathbf{R}\}=M$ is achieved in the case of linear independence of all steering vectors.

\section{B. Subspace Decomposition}

The eigenvalue decomposition of the covariance matrix

$$
\begin{aligned}
\mathbf{R} & =\mathbf{Q} \boldsymbol{\Lambda} \mathbf{Q}^{\mathrm{H}} \\
& =\left[\mathbf{Q}_{\mathrm{s}} \mathbf{Q}_{\mathrm{n}}\right]\left[\begin{array}{cc}
\boldsymbol{\Lambda}_{\mathrm{s}} & \mathbf{0} \\
\mathbf{0} & \boldsymbol{\Lambda}_{\mathrm{n}}
\end{array}\right]\left[\begin{array}{l}
\mathbf{Q}_{\mathrm{S}}^{\mathrm{H}} \\
\mathbf{Q}_{\mathrm{n}}^{\mathrm{H}}
\end{array}\right]
\end{aligned}
$$

leads to a factorisation with a diagonal matrix $\boldsymbol{\Lambda}$ and a unitary modal matrix $\mathbf{Q}$. The eigenvalues in $\boldsymbol{\Lambda}$ split into a noise floor $\boldsymbol{\Lambda}_{\mathrm{n}} \approx \sigma_{v}^{2} \mathbf{I}$ and into a part $\boldsymbol{\Lambda}_{\mathrm{s}} \in \mathbb{R}^{R \times R}$ with eigenvalues above the noise threshold. Thus, the data is know to contain $R$ linearly independent sources which lie in the signal-plus-noise subspace spanned by the columns of $\mathbf{Q}_{\mathrm{s}}$, while $\mathbf{Q}$ spans the noise-only subspace.

\section{Narrowband MUSIC}

When trying to estimate the AoA of sources in $\mathbf{R}$, an idea is to investigate the signal-plus-noise subspace $\mathbf{Q}_{\mathrm{s}}$. However, since $\mathbf{Q}$ is unitary, scanning $\mathbf{Q}_{\mathrm{s}}$ with steering vectors for maxima is likely to extract the steering vector of only the strongest source correctly; otherwise the results will contain orthogonalised basis vectors of the signal subspace in $\mathbf{Q}_{s}$, which are unlikely to match the directions of weaker sources.

Therefore, the idea of the MUSIC algorithm is to scan the noise-only subspace $\mathbf{Q}_{n}$, which is spanned by eigenvectors corresponding to eigenvalues close to the noise floor, $\boldsymbol{\Lambda}_{\mathrm{n}} \approx$ $\sigma_{v}^{2} \mathbf{I}$. The steering vectors of sources that contribute to $\mathbf{R}$ will define the signal-plus-noise subspace $\mathbf{Q}_{\mathrm{S}}$ and therefore lie in the nullspace of its complement $\mathbf{Q}_{n}$. Therefore, the vector $\mathbf{Q}_{n}^{H} \mathbf{a}_{\Omega, \vartheta}$ has to be close to the origin for $\mathbf{a}_{\Omega, \vartheta}$ to be a steering vector of a contributing source. Thus the MUSIC algorithm [5] evaluates its reciprocal,

$$
P_{\mathrm{MU}}(\vartheta)=\frac{1}{\mathbf{a}_{\Omega, \vartheta}^{\mathrm{H}} \mathbf{Q}_{\mathrm{n}} \mathbf{Q}_{n}^{\mathrm{H}} \mathbf{a}_{\Omega, \vartheta}},
$$

with $P_{\mathrm{MU}}(\vartheta)$ also termed the MUSIC spectrum.

\section{Broadband Subspace Decomposition}

\section{A. Space-Time Covariance Matrix}

Different from the narrowband case, in a broadband scenario signal wave fronts travelling across the array at finite speed must be characterised by time delays rather than just phase shifts. This motivates the definition of a polynomial spacetime covariance matrix $\boldsymbol{R}(z) \bullet-\circ \mathbf{R}[\tau]$,

$$
\mathbf{R}[\tau]=\mathcal{E}\left\{\mathbf{x}[n] \mathbf{x}^{\mathrm{H}}[n-\tau]\right\},
$$

which includes the explicit lag value $\tau$. Its $z$-transform $\boldsymbol{R}(z) \bullet-\circ \mathbf{R}[\tau]$ is the cross-spectral density (CSD) matrix, which is parahermitian i.e. $\boldsymbol{R}(z)=\tilde{\boldsymbol{R}}(z)=\boldsymbol{R}^{\mathrm{H}}\left(z^{-1}\right)$.

\section{B. Subspace Decomposition}

The CSD matrix $\boldsymbol{R}(z)$ can be factorised by means of a polynomial eigenvalue decomposition (PEVD) [3], such that

$$
\boldsymbol{R}(z) \approx \boldsymbol{Q}(z) \boldsymbol{\Lambda}(z) \tilde{\boldsymbol{Q}}(z)=\sum_{m=0}^{M-1} \lambda_{m}(z) \boldsymbol{q}_{m}(z) \tilde{\boldsymbol{q}}_{m}(z)
$$

with paraunitary $\boldsymbol{Q}(z)$, i.e. $\boldsymbol{Q}(z) \tilde{\mathbf{Q}}(z)=\tilde{\boldsymbol{Q}}(z) \mathbf{Q}(z)=\mathbf{I}$. The definition of properties is tied to the subband coding problem in [?], which demands $\boldsymbol{\Lambda}(z)$ to be diagonal,

$$
\boldsymbol{\Lambda}(z)=\operatorname{diag}\left\{\Lambda_{0}(z) \Lambda_{1}(z) \quad \ldots \quad \Lambda_{M-1}(z)\right\} \quad,
$$

and spectrally majorised such that power spectral densities $\Lambda_{m}\left(e^{j \Omega}\right)=\left.\Lambda_{m}(z)\right|_{z=e^{j \Omega}}$ fulfil

$$
\Lambda_{m+1}\left(e^{j \Omega}\right) \geq \Lambda_{m}\left(e^{j \Omega}\right) \forall \Omega, m=0 \ldots(M-2) \quad .
$$

Thresholding the polynomial eigenvalues $\Lambda_{m}(z)$ reveals the number of independent broadband sources contributing to 
$\boldsymbol{R}(z)$, and permits a distinction between signal-plus-noise and noise only subspaces,

$$
\boldsymbol{R}(z)=\left[\boldsymbol{Q}_{\mathrm{s}}(z) \boldsymbol{Q}_{\mathrm{n}}(z)\right]\left[\begin{array}{cc}
\boldsymbol{\Lambda}_{\mathrm{s}}(z) & \mathbf{0} \\
\mathbf{0} & \boldsymbol{\Lambda}_{\mathrm{n}}(z)
\end{array}\right]\left[\begin{array}{c}
\boldsymbol{Q}_{\mathrm{s}}^{\mathrm{H}}(z) \\
\boldsymbol{Q}_{\mathrm{n}}^{\mathrm{H}}(z)
\end{array}\right]
$$

similar to the narrowband EVD in (9).

\section{Polynomial MUSIC Algorithm}

The polynomial MUSIC algorithm [1] is an extension of narrowband MUSIC to the broadband case. Similar to the narrowband scenario, P-MUSIC probes the noise-only subspace spanned by the columns of $Q_{\mathrm{n}}(z)$,

$$
\boldsymbol{Q}_{\mathrm{n}}(z)=\left[\begin{array}{lll}
\boldsymbol{q}_{R}(z) & \ldots & \boldsymbol{q}_{M-1}(z)
\end{array}\right] .
$$

The probing requires the definition and implementation of the broadband steering vector in (5) containing fractional delays. One possibility to implement these fractional delays is by means of an appropriately sampled sinc function, such that

$$
a_{m, j}[n]=\operatorname{sinc}\left(n T_{s}-\Delta \tau_{m, j}\right) .
$$

With $A_{m, j}(z) \bullet \longrightarrow a_{m, j}[n]$, a broadband steering vector in the $z$-domain is given by

$$
\boldsymbol{a}_{\vartheta}(z)=\left[\begin{array}{c}
A_{j, 0}(z) \\
\vdots \\
A_{j, M-1}(z)
\end{array}\right] .
$$

The parameter $\vartheta$ on the l.h.s. of (17) indicates the dependency of $\Delta \tau_{j}$ on the AoA. For the implementation of fractional delays in (16), a truncation has to be introduced, leading to an approximation error. More accurate implementations than those based on sampled sinc functions are discussed in [11], [12].

Based on the concept of the narrowband MUSIC algorithm, the generalised quantity

$$
\Gamma_{\vartheta}(z)=\tilde{\boldsymbol{a}}_{\vartheta}(z) \boldsymbol{Q}_{n}(z) \tilde{\boldsymbol{Q}}_{n}(z) \boldsymbol{a}_{\vartheta}(z)
$$

is no longer a norm measuring the vicinity of $\boldsymbol{a}_{\vartheta}(z)$ to the nullspace of $\tilde{\boldsymbol{Q}}_{n}(z)$, but a power spectral density. This has motivated two versions of P-MUSIC algorithm [1] outlined below.

Spatial $P(S P)$-MUSIC. The energy contained in the signal vector $\tilde{\boldsymbol{Q}}_{\mathrm{n}}(z) \boldsymbol{a}_{\vartheta}(z)$ is related to the zero lag term $\gamma_{\vartheta}[0]$ of the auto-correlation-type sequence $\gamma_{\vartheta}[\tau] \circ-\bullet \Gamma_{\vartheta}(z)$ This measure is only dependent on the angle of arrival $\vartheta$, and collects all energy across the spectrum. Instead of searching for the steering vectors providing minimum energy, the reciprocal

$$
P_{S P-M U}(\vartheta)=\frac{1}{\gamma_{\vartheta}[0]} .
$$

is maximised by the angle of arrival $\vartheta$ of signal sources.

Spatio-Spectral P (SSP)-MUSIC. With (18) describing a power spectral density, spectral clues can be exploited in addition to the spatial information extracted by (19). Therefore in addition to spatial localisation of sources,

$$
P_{S S P-M U}(\vartheta, \Omega)=\left(\sum_{\tau=-\infty}^{\infty} \gamma_{\vartheta}[\tau] e^{-j \Omega \tau}\right)^{-1}
$$

can determine over which frequency range sources in the direction defined by the steering vector $\boldsymbol{a}_{\vartheta}(z)$ are active. SP-MUSIC was introduced in [1], but will be omitted from the analysis below, since we will show the impact of the implementation techniques used for PEVD on the performance of SSPMUSIC for both AoA and the frequency ranges of the broadband sources as well, while SP-MUSIC only retrieves AoA information.

\section{ITERATIVE PEVD Algorithms}

Polynomial subspace techniques such as the P-MUSIC algorithm discussed on Sec. IV-C require a polynomial matrix EVD to realise the factorisation of Sec. IV-B. Therefore, this section addresses two iterative algorithms to determine a PEVD and therefore the desired broadband subspace decomposition.

\section{A. Second Order Sequential Best Rotation Algorithm}

The second order sequential best rotation algorithm (SBR2) is an extension of the classical Jacobi algorithm [10] to parahermitian matrices [3]. At each iteration SBR2 finds the maximum element in the parahermitian matrix and transfers its energy onto the diagonal using an elementary paraunitary transformation. The paraunitary transformation includes two operations: first the maximum element is brought onto the zero lag $\mathbf{R}[0]$ with a delay matrix, then the energy from the maximum element is transferred to the diagonal using a Jacobi rotation.

With $\boldsymbol{S}^{(0)}(z)=\boldsymbol{R}(z)$, the $i$ th iteration begins by finding the maximum off-diagonal element based on a set of modified column vectors $\hat{\mathbf{s}}_{k}^{(i)}[\tau] \in \mathbb{C}^{M-1}$, that contain all elements of the $k^{(i)}$ th column of $\mathbf{S}[\tau]$ excluding the element on the diagonal. The lag, $\tau^{(i)}$, and column, $k^{(i)}$ index of the maximum off diagonal element are found using

$$
\left\{k^{(i)}, \tau^{(i)}\right\}=\arg \max _{k, \tau}\left\|\hat{\mathbf{s}}_{k}^{(i-1)}[\tau]\right\|_{\infty} \quad .
$$

Based on $\tau^{(i)}$ and $k^{(i)}$ the maximum element is then delayed onto the zero lag using

$$
\boldsymbol{S}^{(i) \prime}(z)=\tilde{\boldsymbol{\Lambda}}^{(i)}(z) \boldsymbol{S}^{(\boldsymbol{I}-\mathbf{1})}(z) \boldsymbol{\Lambda}^{(i)}(z), \quad i=1 \ldots I,
$$

where

$$
\boldsymbol{\Lambda}^{(i)}=\operatorname{diag}\{\underbrace{1 \ldots 1}_{k^{(i)}-1} z^{-\tau^{(i)}} \underbrace{1 \ldots 1}_{M-k^{(i)}}\}
$$

brings the $k^{(i)}$ th column of $\boldsymbol{S}^{(i-1)}(z)$, shifting it by $\tau^{(i)}$ samples, and $\tilde{\Lambda}^{(i)}$ brings the corresponding $k^{(i)}$ th row onto the zero lag by shifting it in the opposite lag direction.

A Jacobi rotation is then used to eliminate the maximum off-diagonal element, and a unitary matrix $\mathbf{Q}^{(i)}$ applies the Jacobi rotation,

$$
\boldsymbol{S}^{(i)}(z)=\mathbf{Q}^{(i) \mathrm{H}} \boldsymbol{S}^{(i) \prime}(z) \boldsymbol{Q}^{(i)}
$$

The Jacobi rotation affects only two rows and columns of the parahermitian matrix $S^{(i) \prime}(z)$ based on the column and row indices obtained from the maximum off-diagonal element 
search, (21). The energy from the maximum element found using (21) is transferred onto the diagonal with the majority of the energy going to the element which is higher on the diagonal, doing so favours but cannot guarantee spectral majorisation.

Convergence of the SBR2 algorithm has been proven in [3], as the paraunitary operations do not affect the overall energy in the parahermitian matrix, and at each iteration more energy is transferred to the diagonal. The SBR2 algorithm continues either until a fixed number of iterations have elapsed or the maximum off-diagonal element falls below a pre-defined threshold. The delay and rotation matrices can be combined into a single paraunitary matrix,

$$
\boldsymbol{Q}(z)=\prod_{i=1}^{I} \mathbf{Q}^{(i)} \mathbf{\Lambda}^{(i)}(z)
$$

which performs the decomposition according to SBR2.

\section{B. Multiple Shift Maximum Element Sequential Matrix Diag- onalisation}

The multiple shift maximum element sequential matrix diagonalisation algorithm (MSME-SMD) [4] has two major differences compared to the SBR2 method. First, rather than using a simple Jacobi rotation to transfer energy from a single element on the zero lag, as is used in SBR2, MSME-SMD performs a full EVD of the zero lag which clears the energy from all elements in the zero lag matrix $\mathbf{S}^{(i)}[0]$ at each iteration. Also where SBR2 brings a single maximum onto the zero lag during each iteration MSME-SMD aims to shift a total of $(M-1)$ maxima onto the zero lag at each iteration.

In addition to the two major differences mentioned above, the MSME-SMD algorithm also has an initialisation EVD step which ensures that all instantaneous correlations in the parahermitian matrix are removed,

$$
\mathbf{S}^{(0)}[0]=\mathbf{Q}^{(0) \mathrm{H}} \mathbf{R}[0] \mathbf{Q}^{(0)},
$$

where $\mathbf{S}^{(0)}[0]$ is diagonal and the EVD, $\mathbf{Q}^{(0)}$, is applied to all lags of the parahermitian matrix $\boldsymbol{S}^{(0)}(z)=\mathbf{Q}^{(0) \mathrm{H}} \boldsymbol{R}(z) \mathbf{Q}^{(0)}$.

The $i$ th iteration of the MSME-SMD algorithm starts the same way as that of SBR2, using (21) to find the maximum element in the parahermitian matrix $\boldsymbol{S}^{(i)}(z)$. Rather than immediately shifting the energy onto the diagonal as in SBR2, MSME-SMD permutes the first maximum pair into the upper left $2 \times 2$ sub-matrix as shown in Fig. 1(a). Next the search space in Fig. 1(b) is used to find the second maxima, which when brought onto the zero lag will not affect the first maxima in the upper left corner. The second maxima pair is then permuted such that it appears in the upper left $3 \times 3$ sub-matrix. The process is then repeated for the third and fourth maxima using the search spaces shown in Fig. 1(c) and (d). The reduced search spaces shown in Fig. 1 are used to guarantee that a total of $(M-1)$ maxima are brought onto the zero lag at each iteration, by ignoring just the elements the previous maxima appear in only guarantees a total of $(M / 2)$ maxima can be brought onto the zero lag. (a)

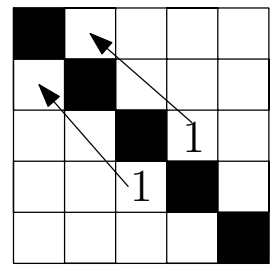

(c)

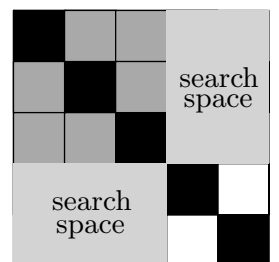

(b)

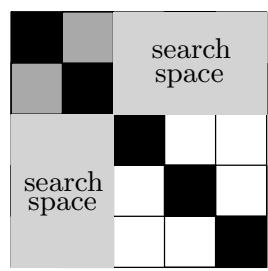

(d)

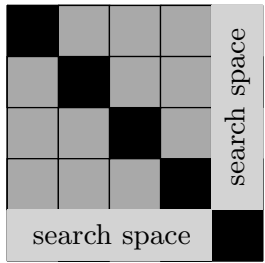

Fig. 1. View of a $5 \times 5$ parahermitian matrix during the $i$ th iteration, not showing the lag dimension: (a) shows the first maxima being permuted into the upper $2 \times 2$ matrix, (b), (c) and (d) show the reduced search spaces used in the $2 \mathrm{nd}$, 3rd and 4th steps of the MSME-SMD serach.

In MSME-SMD the delay matrix $\boldsymbol{\Lambda}^{(i)}$ combines the delay and permutation operations,

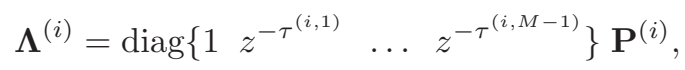

where the $\mathbf{P}^{(i)}$ combines the permutations used to send the maximum elements unto the upper left corner. The lag values used to find the maximum elements $\tau^{(i, m)}, m=1 \ldots(M-1)$ form the delays for each column in (27).

The next step in the $i$ th iteration of MSME-SMD is to diagonalise the zero lag matrix, $\mathbf{S}^{(i) \prime}[0]$, according to (22) however in this case $\mathbf{Q}^{(i)}$ is the modal matrix of an EVD instead of the simple Jacobi rotation used in SBR2.

To finish an iteration of MSME-SMD algorithm, the zero lag is ordered based on the diagonal entries to encourage spectral majorisation. The stopping criteria for MSME-SMD is either a fixed number of iterations or when the maximum off diagonal element falls below a given threshold, identical to that of SBR2. The convergence of the MSME-SMD algorithm is given in [4] along with a more in-depth description of the algorithm and its performance with respect to other PEVD algorithms.

Compared to SBR2 the major advantage of the MSMESMD algorithm is the ability to diagonalise the parahermitian matrix in fewer iterations, in addition the MSME-SMD algorithm can achieve levels of diagonalisation that cannot be achieved using the SBR2 algorithm. The main drawback of the MSME-SMD algorithm is extra computational cost of both the multiple element search and the application of the EVD modal matrix, $\mathbf{Q}^{(i)}$, to all lags.

\section{Simulations AND Results}

The iterative algorithms reviewed in Sec. V achieve different levels of diagonalisation, and therefore different accuracies in the way subspaces are identified. Therefore, below we test the P-MUSIC algorithm as an example for a broadband signal subspace technique to study the impact of diagonalisation, and by implication, the subspace accuracy. In the example below, an $M=8$ element array is illuminated by two broadband 


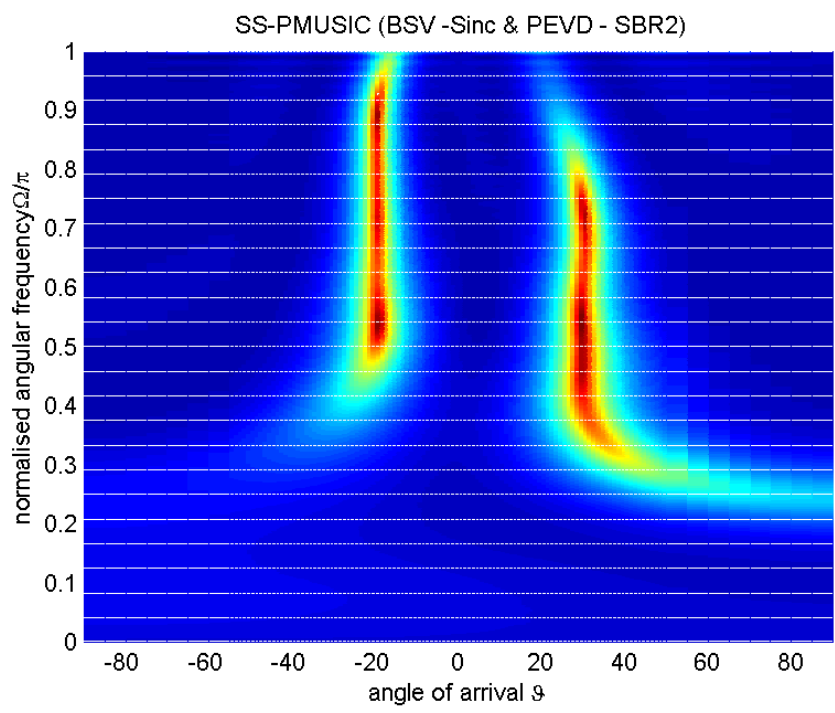

Fig. 2. Performance of SSP-MUSIC based on SBR2 for PEVD for a scenario with two independent broadband sources located at $\vartheta_{1}=-20^{\circ}$ and $\vartheta_{2}=$ $30^{\circ}$ respectively [1].

sources with different AoA and partially overlapping with their spectra:

- source 1 - located at $\vartheta_{1}=-20^{\circ}$, and active over a frequency range $\Omega_{1} \in[0.4688 \pi, 0.9375 \pi]$.

- source 2 - located at $\vartheta_{2}=30^{\circ}$, and active over a frequency range $\Omega_{2} \in[0.3125 \pi, 0.7812 \pi]$.

The array signals are corrupted by uncorrelated independent and identically distributed complex Gaussian noise at $20 \mathrm{~dB}$ SNR. To exclude error sources other than inaccuracies in the subspace identification, we have modelled the data as a sum of closely spaced sinusoids with randomised phases, for whom the individual and highly accurate narrowband steering vectors can be used to simulate the data.

The performance of SSP-MUSIC algorithm with PEVD based on the SBR2 and MSME-SMD approaches are shown in Fig. 2 and 3 respectively, whereby SBR2 achieves a supression of off-diagonal energy down to about $-15 \mathrm{~dB}$, while MSMESMD achieves approximately $-30 \mathrm{~dB}$. The simulations result shows that the SSPMUSIC based on the MSME-SMD decomposition outperform one using SBR2, demonstrating that the accuracy of the EVD is crucial to the performance of any subsequent polynomial subspace-based techniques.

\section{CONCLUSIONS}

This paper has explored the impact of iterative polynomial matrix eigenvalue decompositions - in particular the diagonalisation achieved by these algorithms - on subsequent processing relying on subspace information. Specially, we have studies the impact of an established algorithm called second order sequential best rotation, and compared it to a recent sequential matrix diagonalisation approach with a better suppression of off-diagonal energy in the parahermitian matrix. In simulations of a polynomial MUSIC algorithm for broadband angle of arrival estimation, we have demonstrated that better diagonalisation leads to a better identification of

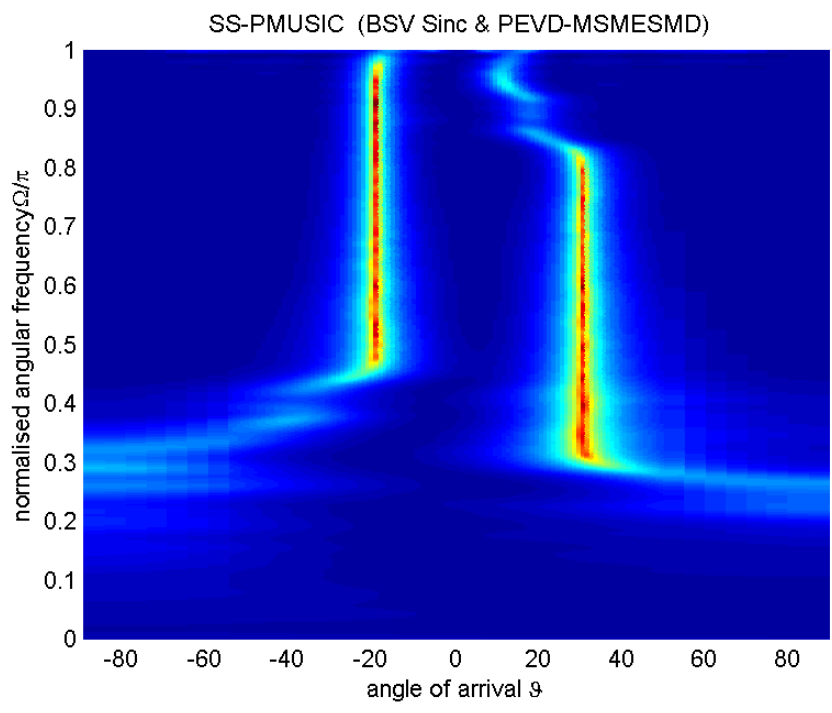

Fig. 3. Performance of SSP-MUSIC based on MSME-SMD for PEVD for a scenario with two independent broadband sources located at $\vartheta_{1}=-20^{\circ}$ and $\vartheta_{2}=30^{\circ}$ respectively.

the relevant signal subspaces, such that e.g. P-MUSIC can extract a cleaner estimate w.r.t. both angle and frequency of the estimated sources.

\section{ACKNOWLEDGEMENT}

This work was supported by the Engineering and Physical Sciences Research Council (EPSRC) Grant number EP/K014307/1 and the MOD University Defence Research Collaboration in Signal Processing.

\section{REFERENCES}

[1] M. Alrmah, S. Weiss, and S. Lambotharan. An extension of the music algorithm to broadband scenarios using polynomial eigenvalue decomposition. In EUSIPCO, Barcelona, Spain, pp. 629-633, Aug. 2011.

[2] J. Dmochowski, J. Benesty, and S. Affes. Direction of arrival estimation using the parameterized spatial correlation matrix. IEEE Trans. Audio, Speech, Lang. Proc., 15(4):1327-1339, May 2007.

[3] J. G. McWhirter, P. D. Baxter, T. Cooper, S. Redif, and J. Foster. An EVD Algorithm for Para-Hermitian Polynomial Matrices. IEEE Trans. Sig. Proc., 55(5):2158-2169, May 2007.

[4] J. Corr, K. Thompson, S. Weiss, J. G. McWhirter, I. K. Proudler Causal Multiple Shift Maximum Element Sequential Matrix Diagonalisation for Parahermitian Matrices. In EUSIPCO, Lisbon, Portugal, Sept. 2014.

[5] R. O. Schmidt. Multiple emitter location and signal parameter estimation. IEEE Trans. Ant. Prop., 34(3):276-280, Mar. 1986.

[6] M. Souden, J. Benesty, and S. Affes. Broadband source localization from an eigenanalysis perspective. IEEE Trans. Audio, Speech, and Lang. Proc., 18(6):1575-1587, Aug. 2010.

[7] H. Hung and M. Kaveh. Focussing matrices for coherent signal-subspace processing. IEEE Trans. ASSP 36(8):1272-1281, Aug. 1988.

[8] P. Vaidyanathan. Theory of optimal orthonormal subband coders. IEEE Trans SP, 46(6):1528-1543, June 1998.

[9] P. Pal and P. P. Vaidyanathan. A novel autofocusing approach for estimating directions-of-arrival of wideband signals. In 43rd Asilomar Conf. SSC, pp:1663-1667, Pacific Grove, CA, Nov. 2009.

[10] G. H. Golub and C. F. Van Loan. Matrix Computations. John Hopkins University Press, 3rd ed., 1996.

[11] M. Alrmah and S. Weiss. Filter Bank Based Fractional Delay Filter Implementation for Widely Accurate Broadband Steering Vectors. In CAMSAP, St. Martin, Dec. 2013.

[12] M. Alrmah, S. Weiss, and J.G. McWhirter. Implementation of Accurate Broadband Steering Vectors for Broadband Angle of Arrival Estimation. IET Int. Sig. Proc., Dec. 2013. 